\title{
AMS SPRING SECTIONAL SAMPLER
}

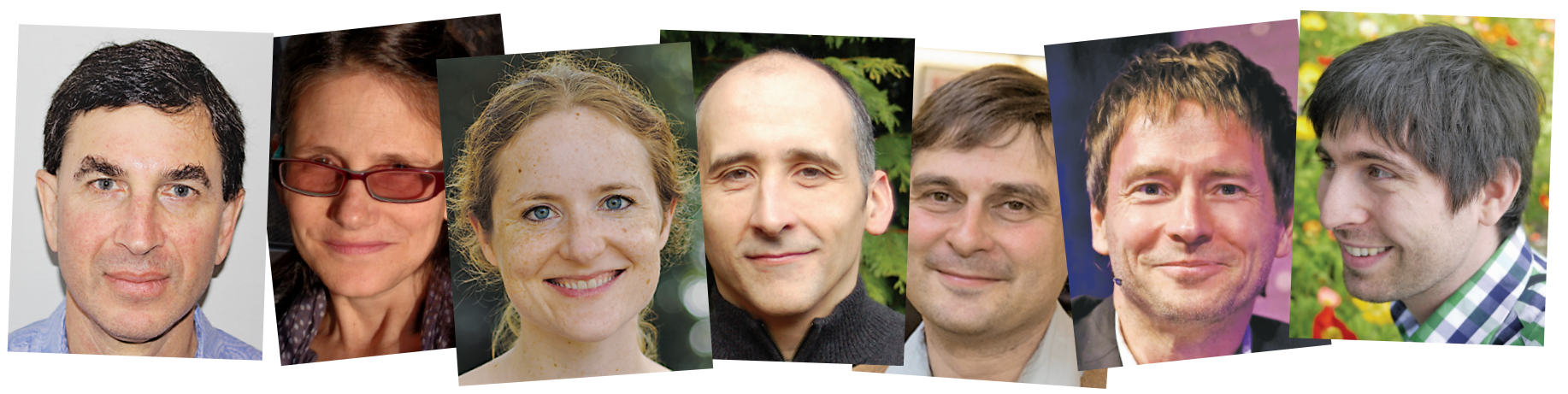

From left to right: J. M. Landsberg, Jennifer Morse, Kirsten Wickelgren, Sándor Kovács, Dimitri Shlyakhtenko, Edward Frenkel, and Valentino Tosatti.

In this sampler, the speakers below have kindly provided introductions to their Invited Addresses for the AMS Spring Southeastern Sectional (Vanderbilt University, April 14-15), the AMS Spring Western Sectional (Portland State University, April 14-15), and the AMS Spring Eastern Sectional (Northeastern University, April 21-22) Meetings.

\section{Spring Southeastern Sectional Meeting}

On the Geometry of Matrix Multiplication

by J. M. Landsberg

page 402

Computing, Combinatorics, and $k$-Schur Functions

by Jennifer Morse

page 403

An Arithmetic Count of the Lines

on a Smooth Cubic Surface

by Kirsten Wickelgren

page 404

\section{Spring Western Sectional Meeting}

Moduli Theory and Singularities

by Sándor Kovács

page 406

A (Co)homology Theory for Subfactors and Planar Algebras

by Dimitri Shlyakhtenko

page 408

\section{Spring Eastern Sectional Meeting}

AMS Einstein Public Lecture:

Imagination and Knowledge

by Edward Frenkel

page 410

Metric Limits of Calabi-Yau Manifolds

by Valentino Tosatti

page 413

For permission to reprint this article, please contact: reprint-permission@ams . org.

DOI: http://dx.doi.org/10.1090/noti1656 


\section{J. M. Landsberg}

\section{On the Geometry of Matrix Multiplication}

Our story begins with a spectacular failure: The standard algorithm to multiply two $n \times n$ matrices uses $n^{3}$ multiplications. In 1969, while attempting to show that the standard algorithm was optimal, V. Strassen discovered an explicit algorithm to multiply $2 \times 2$ matrices using 7 multiplications rather than $8=2^{3}$.

Strassen's algorithm may also be used to multiply $n \times n$ matrices using $\mathcal{O}\left(n^{2.81}\right)$ arithmetic operations instead of the usual $\mathcal{O}\left(n^{3}\right)$. Subsequent work steadily lowered $\mathcal{O}\left(n^{2.81}\right)$ to $\mathcal{O}\left(n^{2.38}\right)$ and has led to the astounding conjecture that asymptotically it is essentially just as easy to multiply matrices as it is to add them. More precisely, it has been conjectured that for any $\epsilon>0$, the multiplication of $n \times n$ matrices can be performed using

The exponent

of matrix multiplication

is a

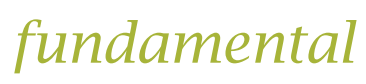

constant. $\mathcal{O}\left(n^{2+\epsilon}\right)$ arithmetic operations. It is a central question to determine just how efficiently one can multiply $n \times n$ matrices, both practically and asymptotically. The exponent $\omega$ of matrix multiplication is defined to be the infimum over $\tau$ such that $n \times n$ matrices can be multiplied using $\mathcal{O}\left(n^{\tau}\right)$ arithmetic operations. The exponent is a fundamental constant governing the complexity of all operations in linear algebra, and there has been considerable effort to determine or at least bound it. Regarding practical matters, Strassen's algorithm is practical when large $(n \geq 2,000)$ unstructured matrices are multiplied. Given the central role of matrix multiplication in all computation, any new, efficient, practical algorithm would have dramatic impact.

In this talk, I will present a history of the problem, both of upper and lower complexity bounds. I will discuss how geometry, more precisely algebraic geometry and representation theory, are used. In particular, I will explain how, had someone asked him one hundred years ago, the algebraic geometer Terracini could have predicted Strassen's algorithm. The talk will conclude with the recent use of representation theory to construct algorithms, more precisely, rank decompositions.

In particular I will discuss Figure 1, which arises in a new algorithm for $3 \times 3$ matrices discovered by my PhD student Austin Conner.

For those who can't wait for the talk, a detailed history and the state of the art appears in [1].

J. M. Landsberg is professor of mathematics at Texas A\&M University. His email address is jm1@math. tamu. edu.

For permission to reprint this article, please contact:

reprint-permission@ams .org.

DOI: http://dx.doi.org/10.1090/noti1659

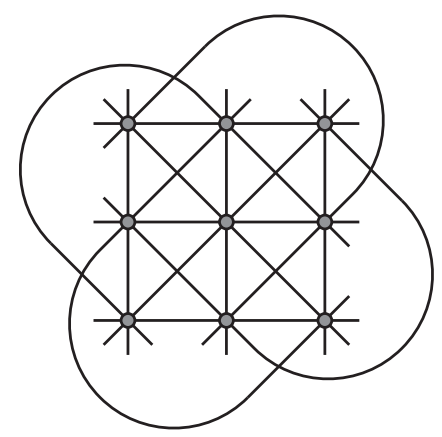

Figure 1. My PhD student Austin Conner discovered a new algorithm for $3 \times 3$ matrices.

\section{References}

[1] J. M. LAnDSBerg, Geometry and Complexity Theory, Cambridge Studies in Advanced Mathematics, vol. 169, Cambridge Univ. Press, 2017.

\section{Image Credit}

Figure 1 Hesse configuration by David Eppstein (2012), reproduced under the Creative Commons Attribution-ShareAlike License.

Author photo courtesy of J. M. Landsberg.

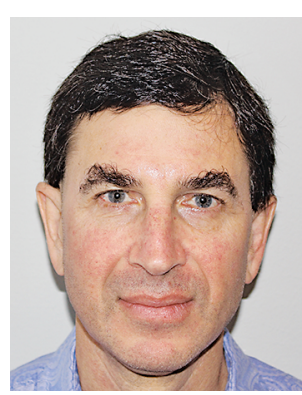

J. M. Landsberg

\section{ABOUT THE AUTHOR}

J. M. Landsberg is professor of mathematics at Texas A\&M University. His research interests include differential geometry, algebraic geometry, representation theory, and complexity theory. He coorganized a fall 2014 semester on geometry and complexity at the Simons Institute for Theoretical Computing, where he also served as a UC Berkeley Chancellor's professor. 


\section{Jennifer Morse}

\section{Computing, Combinatorics, and $k$-Schur Functions}

Combinatorial structures can be used to understand symmetric functions such as Macdonald polynomials and $k$-Schur functions and to attack long-standing open problems in algebraic combinatorics.

Shortly after undergraduate school, I found myself living in a condemned house in San Diego with one of America's most wanted killers and tutoring surfers to scrape by. One afternoon I saw a student sitting in a cafe scribbling frantically over scraps of paper filled with mysterious diagrams (Figure 1) and I was intrigued. When

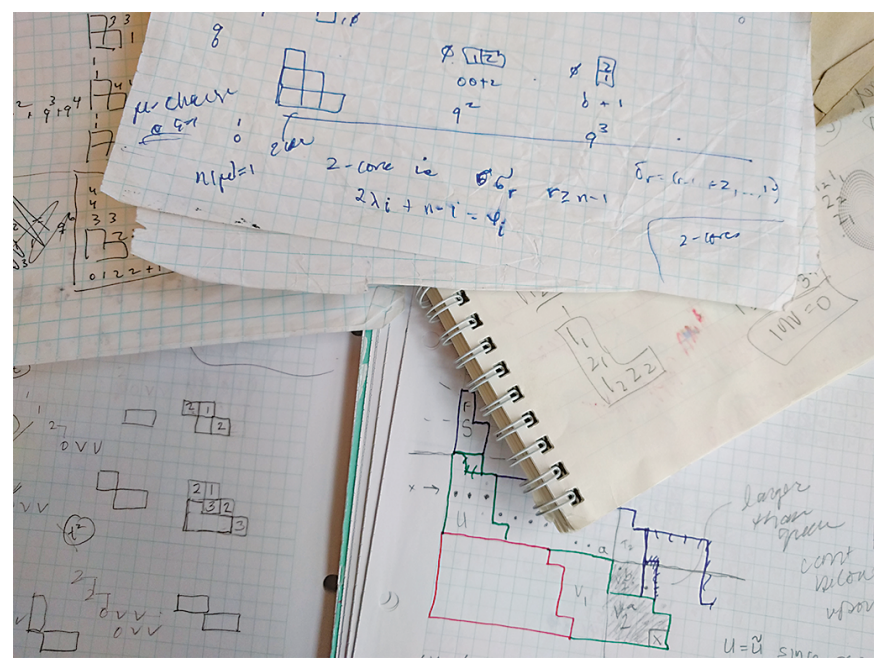

Figure 1. Diagrams which illustrate how to multiply symmetric polynomials also apply to enumerative geometry and representation theory.

I was told not only that the diagrams capture a rule for multiplying polynomials but that playing with these magical combinatorial objects constituted a living and came with subsidized housing, I was ready to sign up. The polynomials are multivariate and have the property of being invariant under any permutation of their variables. For example,

$$
3 x_{1}+3 x_{2}+3 x_{3}-17 x_{1} x_{2}-17 x_{1} x_{3}-17 x_{2} x_{3}
$$

has this symmetry property, whereas

$$
3 x_{1}+5 x_{2}+3 x_{3}-17 x_{1} x_{2}-17 x_{1} x_{2}-17 x_{2} x_{3}
$$

Jennifer Morse is professor of mathematics at the University of Virginia. Her email address is morsej@vi rginia. edu.

For permission to reprint this article, please contact: reprint-permission@ams.org.

DOI: http://dx.doi.org/10.1090/noti1668
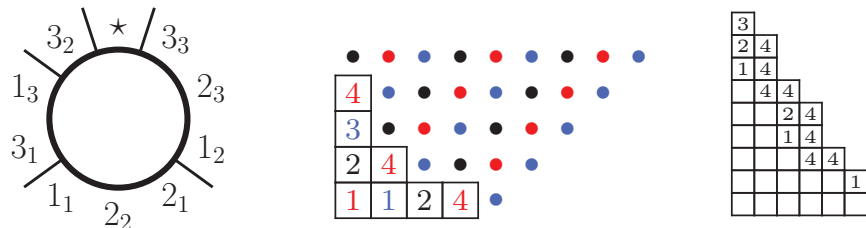

Figure 2. Combinatorial structures beautifully characterize $k$-Schur functions and Macdonald polynomials.

does not. The student's spiel at the cafe included a defensive explanation that the same rule for computing products of these symmetric polynomials is used to determine the structure of the cohomology of the variety of $r$-dimensional subspaces of $\mathbb{C}^{n}$ and the decomposition of Young modules into irreducible representations. Although my eyes glazed over at the time, the connection between polynomial computation and problems in enumerative geometry and representation theory soon became my personal obsession.

Now, the line of research is on other distinguished families of multivariate polynomials which carry information of more contemporary interest. A notable example is the Macdonald polynomials, symmetric functions which arise in the wave equations of certain systems of quantum relativistic particles on a circle and as the Frobenius images of bigraded Garsia-Haiman modules. As with the example discussed at the cafe, key information is wrapped up in products of the distinguished families or in their coefficients when decomposed into more classical symmetric function bases. However, the computations are now extremely intricate, and efficient rules are notoriously hard to come by. The challenge of finding a combinatorial structure to naturally facilitate computation is a central theme in algebraic combinatorics.

This was my focus in the late 1990s when Alain Lascoux, Luc Lapointe, and I uncovered the $k$-Schur functions. These are subfamilies of symmetric functions which can be used to reflect the structure of quantum cohomology: products of $k$-Schur functions generate Gromov-Witten invariants for the flag variety. My passion has since been to find and develop the combinatorics necessary for computing with $k$-Schur functions. It has been exciting to find that a marriage of Young tableaux and the Bruhat order on the type- $A$ affine Weyl group beautifully characterizes $k$-Schur functions (Figure 2). When my 'tween daughter scowls at me for having the gall to serve fish, when my son throws himself to the floor of a city bus howling for no apparent reason, or when I miss the deadline to submit my blurb to the Notices, I am calmed by the image of a combinatorial creation and by thinking about just how quickly we can now generate the associated symmetric polynomials.

I look forward to sharing details about Macdonald polynomials and $k$-Schur functions. We will discuss related long-standing open problems in algebraic combinatorics 


\section{AMS SPRING SECTIONAL SAMPLER}

as food for thought during breaks. Seeing a problem unfold with simple diagrams after banging my head against it for twenty years makes it hard to imagine doing anything else.

\section{Image Credits}

Author photo by Scarlett Morse.

All other images courtesy of Jennifer Morse.

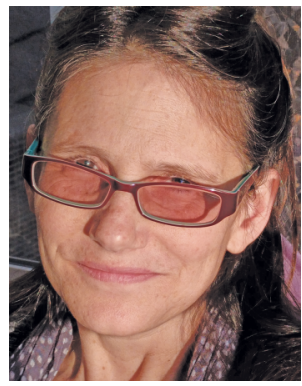

Jennifer Morse

\section{ABOUT THE AUTHOR}

Jennifer Morse was named a Simons Fellow for her work in algebraic combinatorics on $k$-Schur functions; these symmetric functions were the theme of "Affine Schubert Calculus: Combinatorial, Geometric, Physical, and Computational Aspects," a Focused Research Grant supported by the National Science Foundation.

\section{Kirsten Wickelgren}

\section{An Arithmetic Count of the Lines on a Smooth Cubic Surface}

There are 27 lines on the complex Fermat cubic surface

$$
\left\{\left(x_{1}, x_{2}, x_{3}\right) \in \mathbb{C}^{3}: x_{1}^{3}+x_{2}^{3}+x_{3}^{3}=-1\right\} .
$$

It's not hard to write down these lines: let $x_{1}$ be a fixed third root of -1 ; let $x_{2}=\zeta x_{3}$, where $\zeta^{3}=-1$ is another third root of -1 ; and let $x_{3}$ vary arbitrarily, thereby parametrizing a line. Changing the roots of -1 and which coordinate gets fixed gives $3 \times 3 \times 3=27$ lines. Now, when the two chosen roots of -1 are both -1 itself, the resulting line has all real coefficients and therefore also determines a line on the real surface (shown in Figure 1). There are three such lines, and they all have the property that as you travel down the line, the rotation of the tangent plane around the line changes direction and never makes a full spin around the axis.

It is a celebrated nineteenth-century result due to Salmon and Cayley that the number of lines on any smooth cubic surface over the complex numbers is 27 ; so in particular, this number does not depend on the surface. This is no longer true over $\mathbb{R}$ : Shläfli classified smooth real cubic surfaces by their number of real lines and tritangent planes, and there can be $3,7,15$, or 27 real lines. Extensive work has been done classifying cubic surfaces. Over the real numbers, Segre distinguished between two types of real lines: If the tangent planes to the surface along a line do not spin all the way around, the line is called hyperbolic; otherwise it is elliptic. It follows from work of Segre that the number of hyperbolic minus the number of elliptic lines is always 3 . In other words, although the count of the number of lines varies, there is a signed count that is independent of the surface. This invariant number 3 does not seem to have been explicitly remarked upon until recent work of OkonekTeleman, Finsahin-Kharlamov, and Benedetti-Silhol, who also provide proofs that do not rely on an exhaustive classification. These modern proofs are topological. The first two use characteristic classes on real Grassmannians and compute that a sign in a local contribution from a line changes depending on whether the line is elliptic or hyperbolic.

$\mathbb{A}^{1}$-homotopy theory was introduced in the late $1990 \mathrm{~s}$ by Fabien Morel and Vladimir Voevodsky. It provides tools to do algebraic topology with spaces replaced by polynomial equations themselves or, more precisely, to do algebraic topology with schemes. Instead of using

Kirsten Wickelgren is assistant professor at the Georgia Institute of Technology, supported by National Science Foundation Award DMS-1406380. Her email address is kwicke1gren3@math.gatech.edu.

For permission to reprint this article, please contact: reprint-permission@ams . org.

DOI: http://dx.doi.org/10.1090/noti1663 


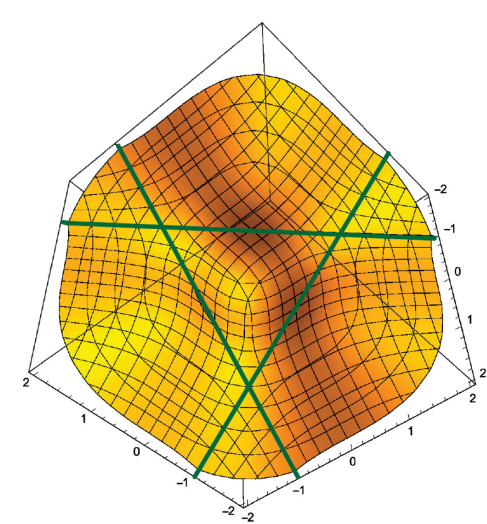

Figure 1. The real Fermat Cubic Surface $\left\{\left(x_{1}, x_{2}, x_{3}\right) \in \mathbb{R}^{3}: x_{1}^{3}+x_{2}^{3}+x_{3}^{3}=-1\right\}$ contains the three green lines.

topological constructions over real points, one can use $\mathbb{A}^{1}$ homotopy theory over any field. Moreover, information about the arithmetic of the schemes is automatically recorded in $\mathbb{A}^{1}$-homotopy theory's analogues of classical topological tools. In my talk at the AMS Southeastern Sectional Meeting, I'll discuss joint work with Jesse Leo Kass giving a count of the lines on a smooth cubic surface over an arbitrary field of characteristic not 2 which records information about the arithmetic of the lines.

It is a feature of $\mathbb{A}^{1}$-homotopy theory that certain invariants that are elements of $\mathbb{Z}$ in classical algebraic topology now lie in a group of formal differences of quadratic forms due to Witt. Taking the quadratic form to its rank then recovers the classical count over $\mathbb{Z}$. But a quadratic form carries more data than its rank, and applying other invariants recovers new equalities. Aravind Asok, Jean Fasel, Marc Hoyois, Jesse Kass, and Marc Levine, and I have done recent work along these lines in various contexts.

It is a consequence of joint work with Kass that there is a fixed quadratic form (I'll tell you which one in the talk!) that equals a sum over the lines in a cubic surface of a weight determined by arithmetic data associated to the line. This arithmetic data consists of the field generated by the coefficients of the equations defining the line and the behavior of the tangent planes along that line. A concrete consequence is the following: for a smooth cubic surface over a finite field, the number of elliptic lines is even, provided the finite field is chosen to be large enough so that it contains all the coefficients defining the lines.

\section{Image Credits}

Figure 1 courtesy of Kirsten Wickelgren. Author photo courtesy of Joe Rabinoff.

\section{ABOUT THE AUTHOR}

Kirsten Wickelgren's research is in homotopy theory and arithmetic geometry; she especially likes Grothendieck's anabelian program. ${ }^{1}$ Her one-year-old likes to wear gloves on his feet.

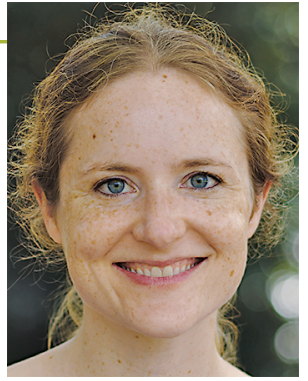

Kirsten Wickelgren

\footnotetext{
${ }^{1}$ See her "WHAT IS...an Anabelian Scheme" in the March 2016 Notices, www. ams.org/pub7ications/journals/ notices /201603/rnoti-p285.pdf.
} 


\section{AMS SPRING SECTIONAL SAMPLER}

\section{Sándor Kovács}

\section{Moduli Theory and Singularities}

In this talk I will discuss recent advances in the moduli theory of higher-dimensional algebraic varieties. Of course, half of the words in that sentence merit explanation, so that will be included in the talk as well. Some of these advances concern the singularities that appear on stable varieties and their influence on the geometry of the corresponding moduli spaces...yet more words to explain.

The roots of moduli theory can be traced to a short remark of Bernhard Riemann in his 1857 treatise on abelian functions (nowadays we would say compact Riemann surfaces). This remark suggests that the space of equivalence classes of compact complex Riemann surfaces of genus $g>1$ can be parametrized by $3 g-3$ complex parameters, which Riemann called moduli.

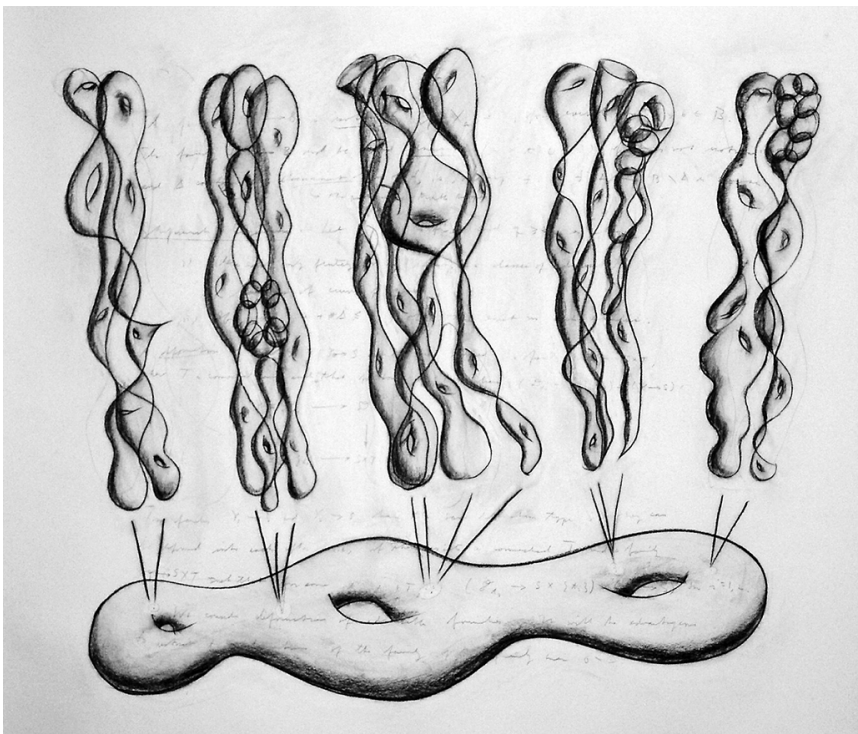

Figure 1. Riemann's $3 g-3$ dimensional moduli space of compact Riemann surfaces of genus $g$.

In other words, fix a compact connected orientable (topological) surface of genus $g$ and consider the various ways one can equip it with a complex structure. According to this remark of Riemann, the possible complex structures can be described by $3 g-3$ parameters. Or, in modern language, the space of those complex structures is $3 g-3$-dimensional. After Riemann, these spaces are called moduli spaces.

Sándor Kovács is the Craig McKibben and Sarah Merner Professor of Mathematics at the University of Washington. His email address is skovacs@uw.edu.

For permission to reprint this article, please contact: reprint-permission@ams.org.

DOI: http://dx.doi.org/10.1090/noti1655
Lars Ahlfors, one of the 1936 Fields medalists and one of the first to embark on the quest to make Riemann's remark rigorous, said in his 1962 ICM address: “Riemann's classical problem of moduli is not a problem with a single aim, but rather a program to obtain maximum information about a whole complex of questions which can be viewed from several different angles."

An algebraic approach to moduli spaces was pioneered by David Mumford, a 1974 Fields Medalist. When viewed algebraically over the complex numbers, a Riemann surface is one-dimensional. Hence it is called an algebraic curve. Taking advantage of the algebraic point of view, Mumford extended the moduli problem to include degenerations of these algebraic curves, i.e., Riemann surfaces with singularities (as in Figure 2). This is a delicate matter, as allowing arbitrary singularities would lead to an intractable problem. In contrast, it is possible to improve the singularities of the degenerate fibers without changing the smooth fibers of a given family of curves. For example, consider the family of degree 5 plane curves with equations $x^{5}-y^{2}+t\left(5 x^{3}-4 x-4\right)=0$ (as in Figure 3 ), parametrized by $t$. As long as $t \neq 0$, the above equation defines a smooth algebraic curve, but for $t=0$ the defined curve, $x^{5}-y^{2}=0$ (denoted by red in Figure 3), is singular. However, this degeneration can be improved considerably by making a change of variables given by $y=x^{2} z$. This will not affect the smooth members of the family, i.e., the ones with $t \neq 0$, but will replace the singular member by the curve defined by the equation $x^{4}\left(x-z^{2}\right)=0$. Making another change of variables given by $x=z w$ leads to the equation $z^{5} w^{4}(z-w)$. The curve defined by this equation is the union of three lines, much simpler than the original singular degeneration.

\section{Stable singularities in higher dimensions are much more diverse complicated.}

Mumford realized that a similar process can always be used to improve the singularities of the degenerate fibers. This led to the definition of stable curves, which are algebraic curves whose only allowable singularities are transversal intersections of smooth branches, such as in the curve in Figure 4 defined by the equation $y^{2}=$ $x^{2}(x+1)$. Note that this picture only shows the real points of this curve. All complex points and the true topology of this curve are shown by the green complex curve on the right-hand side in Figure 2.

In addition to giving an algebraic construction for the moduli space of smooth algebraic curves, Mumford also succeeded in constructing a moduli space for stable curves and proving that this moduli space can be equipped with the structure of a projective variety with the moduli space of smooth algebraic curves as a dense open set. 

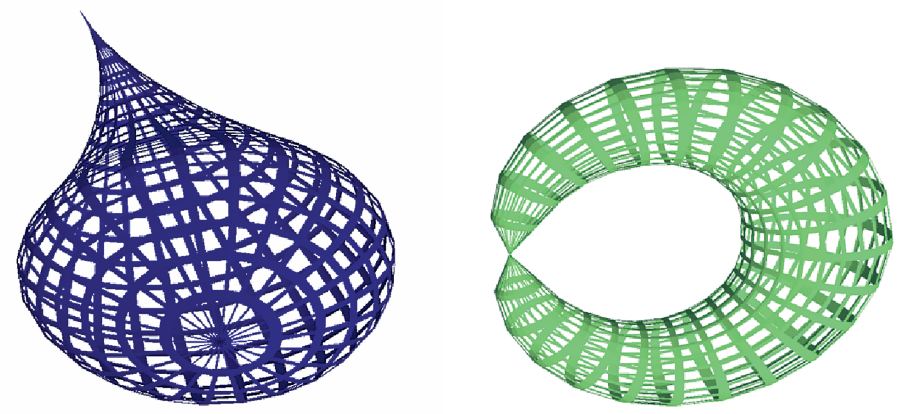

Figure 2. Mumford extended the moduli problem to include Riemann surfaces with singularities.

Ever since Mumford's seminal work, many properties of these moduli spaces have been studied. The various applications discovered are so numerous and broad ranging that it would be impossible to list them in a concise manner. In fact, several disciplines grew out of the study of moduli problems, as shown by the number of MSC classification categories devoted to such disciplines.

Naturally, the question arises whether something similar is possible for higher-dimensional varieties as in

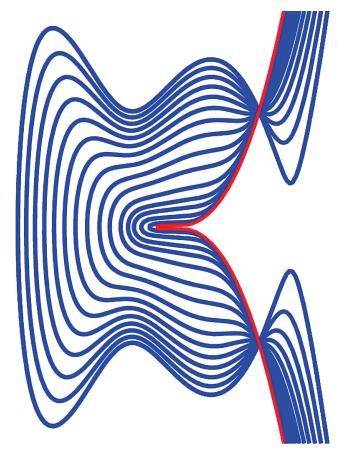

Figure 3. This family of plane curves can be reparametrized to make the components of the singular red one smooth.

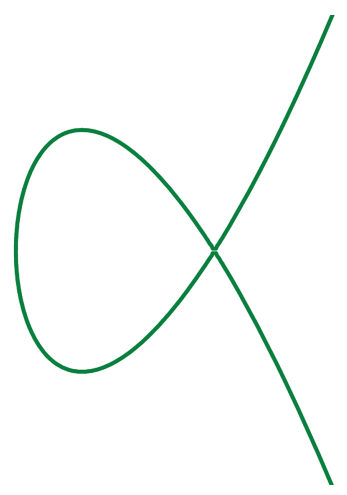

Figure 4. A stable curve, such as $y^{2}=x^{2}(x+1)$, is one with no singularities except for transversal selfintersections.

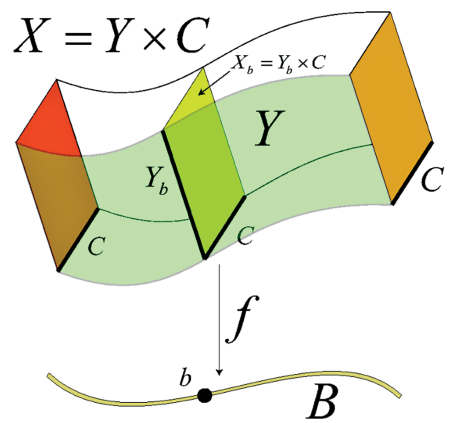

Figure 5. Constructing moduli spaces of higher dimensional varieties leads to new challenges.

Figure 5. Interestingly, this question was not answered satisfactorily until recently, and to some extent it is still not answered completely. There are several reasons for this. While the singularities of stable curves are the most simple curve singularities-the transversal intersection of two smooth branches-stable singularities in higher dimensions are much more diverse and complicated. The families considered in the moduli problem are also more complicated. A stable family of curves is simply a family of stable curves, but this is no longer true in higher dimensions. A stable family, beyond being a family of stable objects, has further properties which reflect conditions on the family, not only on its fibers. In this talk I will discuss these intriguing issues along with the most recent related results. 


\section{Image Credits}

Figure 1, courtesy of Lun-Yi Tsai, shows Lun-Yi Tsai's Shafarevich's Conjecture, Tunyitsai.com/demonstrations /co11aborations.htm.

Figures 2-5, and author photo courtesy of Sándor Kovács.

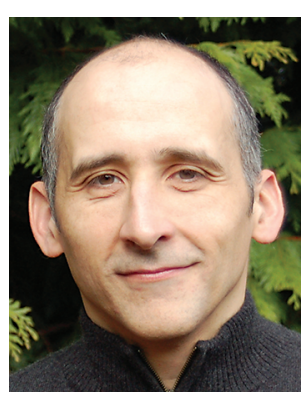

\section{ABOUT THE AUTHOR}

When Sándor Kovács is not thinking about algebraic geometry, he enjoys swimming, biking, running, and hiking. One of his current goals is to improve his butterfly technique. He is also working toward a perfect headstand.

\section{Dimitri Shlyakhtenko}

\section{A (Co)homology Theory for Subfactors and Planar Algebras}

I am very grateful to be speaking on my joint work with S. Vaes and S. Popa [3].

I would like to start with a construction that a priori has nothing to do with subfactors or planar algebras. Let $S_{n}^{2}$ denote the two-sphere with $n+1$ distinct points $p_{1}, \ldots, p_{n+1}$ removed and let $\delta \geq 2$ be a fixed real number. Let $V_{n}$ be the linear space whose basis consists of isotopy classes of zero or more closed curves drawn on $S_{n}^{2}$, subject to the relation that if a curve bounds a disk that does not include any other curves or the points $p_{j}$, then the curve can be removed up to a multiplicative factor $\delta$. Figure 1 shows two equalities of diagrams on $S_{2}^{2}$. The equalities are due to the relation $\bigcirc=\delta$ and the fact that the drawing is on a sphere.
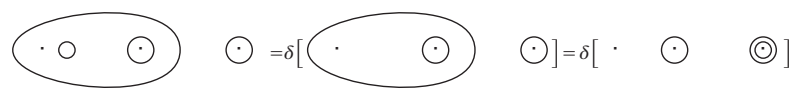

Figure 1. Removing a trivial curve is equivalent to multiplication by $\delta$. The second equality holds because the drawing is on a sphere.

We define a differential complex structure on $\left(V_{n}\right)_{n \geq 0}$. Let $\alpha_{j}^{(n)}: S_{n}^{2} \rightarrow S_{n-1}^{2}$ be the map in which the point $p_{j}$ is glued back into $S_{n}^{2}$ and the remaining points are renumbered (in order) as $p_{1}, \ldots, p_{n}$. Let $\epsilon_{j}^{(n)}: V_{n} \rightarrow V_{n-1}$ be the map in which a collection of curves on $S_{n}^{2}$ is redrawn on $S_{n-1}^{2}$ via the map $\alpha_{j}^{(n)}$. Then $\partial_{n}=\sum(-1)^{k} \epsilon_{k}^{(n)}$ satisfies $\partial_{n} \circ \partial_{n+1}=0$, and so we can define a sequence of homology spaces $H_{n}=\operatorname{ker} \partial_{n} / \operatorname{im} \partial_{n+1}$.

The next challenge is to compute these spaces. One is tempted to do this "by hand"; indeed for $n=0,1,2$ one can easily describe all arrangements of curves on $S_{n}^{2}$. Some amount of computation then shows that $H_{0}=\mathbb{C}$ and $H_{1}=H_{2}=0$. However, for $n \geq 3$ things get complicated, and I am actually not aware of an easy combinatorial computation, even of $\mathrm{H}_{3}$.

To compute $H_{n}$ we need to reinterpret them by redrawing and making apparent the connection with Temperley-Lieb-Jones diagrams; see Figure 2.

Leaving Temperley-Lieb-Jones diagrams aside for the moment, let us consider what happens if we permit other types of elements $x$ as in Figure 2. Suppose that $G$ is a group with a finite generating set $X$. For each element

Dimitri Shlyakhtenko is professor of mathematics at UCLA. His email address is sh7yakht@math.ucla.edu.

For permission to reprint this article, please contact: reprint-permission@ams .org.

DOI: http://dx.doi.org/10.1090/noti1662 


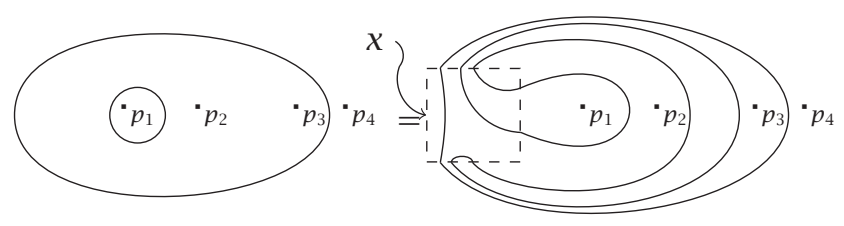

Figure 2. Any diagram can be redrawn to ensure that the punctures are surrounded by parallel arcs of circles, while all of the complexity of the diagram is isolated in a small region $x$. Note that $x$ ends up being what is called a Temperley-Lieb-Jones diagram: a planar diagram in which an even number of points along the boundary of a square are connected in a noncrossing way by curves.

$g \in G$, let us choose a way to write $g=g_{1} \cdots g_{m}, g_{j} \in X$, and consider as in [1]

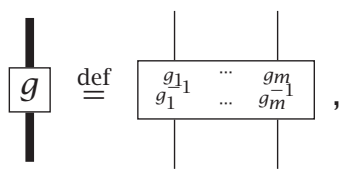

with relations: $\frac{g}{g}=\underline{g h}$ and $\bigcirc g=1$.

Here thick lines represent multiple parallel strings. Although a diagram $g$ depends on how we represented $g \in G$ as a product of generators, if we paste such a diagram onto the surface of $S_{n}^{2}$ in such a way that top and bottom strings are connected to each other, this dependence disappears.

The vector spaces spanned by classes of such diagrams drawn on $S_{n}^{2}$ again form a differential complex. A little thought shows that the map given by

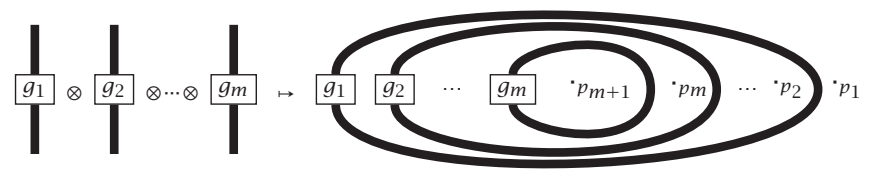

is an isomorphism between the bar complex defining the group homology $H_{*}(G ; \mathbb{C})$ and our differential complex! This strongly suggests the original homology spaces $H_{k}$ defined by curves on $S_{n}^{2}$ have an interpretation as a kind of group homology.

This is indeed the case, but a group has to be replaced by a quantum symmetry encoded in a planar algebra introduced by Jones in the context of his subfactor theory. There is a nice object coming from subfactor theory, called the tube algebra, and it turns out that the homology groups we discussed at the start of this article precisely compute its Hochschild homology. The picture becomes complete when its turns out that, due to some Morita equivalence, our homology is the same as the homology of a certain quasinormal inclusion of two von Neumann algebras. In this generality, we obtain a common way of viewing group homology, as well as homology for ergodic equivalence relations (including $L^{2}$ homology of Gaboriau), and homology for $C^{*}$-tensor categories. This also leads to a computation answering the question at the start of this paper. Using the connection between Temperley-Lieb-Jones diagrams and the representation category of $S U_{q}(2)$ (here $\delta=q+q^{-1}$ ) Kyed et al. [2] have recently used Bichon's three-dimensional projective resolution for this representation category to conclude that $H_{3}=\mathbb{C}$ and $H_{n}=0$ for $n \notin\{0,3\}$.

\section{References}

[1] V. F. R. Jones, Planar Algebras, preprint, Berkeley, 1999, arXiv:math.QA/9909027.

[2] D. Kyed, S. RAuM, S. VAes, and M. VAlveKens, $L^{2}$-Betti numbers of rigid $C^{*}$-tensor categories and discrete quantum groups, Anal. PDE 10 (2017), 1757-1791. MR3683927

[3] S. POPA, D. SHLYAKHTENKo, and S. VAES, Cohomology and $L^{2}$ Betti numbers for subfactors and quasi-regular inclusions, to appear in Int. Math. Res. Not.

\section{Image Credits}

All figures courtesy of author.

Author photo courtesy of Reed Hutchinson/UCLA.

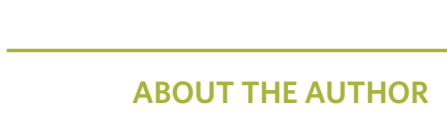

Dimitri Shlyakhtenko's research focuses on free probability and its applications to operator algebras, random matrices, and subfactor theory. He is currently serving as the director of the Institute for Pure and Applied Mathematics (IPAM).

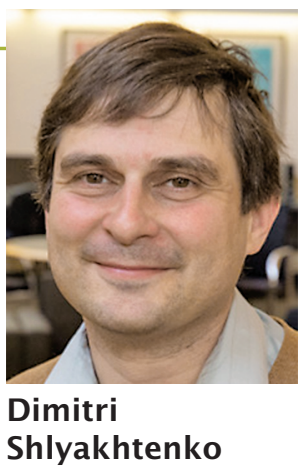




\section{AMS SPRING SECTIONAL SAMPLER}

\section{Edward Frenkel}

\section{AMS Einstein Public Lecture: Imagination and Knowledge}

It is an honor to give the AMS Einstein Public Lecture this year. The title of my lecture is "Imagination and Knowledge." As this is a public lecture, I would like to talk about the essence of our profession, its role in today's world, and the responsibilities that come with it. We live in the age of artificial intelligence, with mathdriven information technology invading our lives at an accelerating pace, bringing us new opportunities and unprecedented connectivity but also multiple challenges to our sense of identity and reality, our culture, and even the truth. It's not surprising that in this environment many people look to mathematicians, expecting us to provide some clarity and perhaps even some guidance on how to navigate today's world. For example, a famous author recently asked me, "Is life just an algorithm?" In fact, that's what I often hear, even from some super-smart folks: life is an algorithm; love: just a chemical reaction; a human: nothing but a sequence of 0 s and $1 \mathrm{~s}$. But is it really true? That's what I want to talk about in this lecture.

I want to start with a quote from Albert Einstein: "Imagination is more important than knowledge. For knowledge is limited, whereas imagination embraces the entire world." In my talk, I will give examples from the history of mathematics that support Einstein's view. These examples show how imagination provides bursts of insight that enable mathematicians to make new advances and to abandon what was taken for granted as well known and well understood.

My first example is the discovery of imaginary numbers (no pun intended!) in Gerolamo Cardano's wonderful treatise Ars Magna (circa 1545, Figure 1). At the time, people thought that the square root of a negative number could not possibly exist. How could it, if the square of any real number is always nonnegative? Everybody knew that, case closed. But Cardano did not take this "knowledge" for granted. Instead, he dared to imagine that such numbers existed and tried to use them (specifically, the square root of -15 ) to tackle mathematical problems he was interested in. And though in the process of doing it he endured, in his own words, "mental tortures", he did succeed, proving that the idea was viable. Thus, imaginary numbers were born. Today, we cannot imagine our life without them. Moreover, they are not just a clever trick or a tool. They are essential elements of quantum mechanics, describing the fundamental structure of physical reality.

Edward Frenkel is professor of mathematics at the University of California, Berkeley. His email address is frenke1@math . berkeley . edu.

For permission to reprint this article, please contact:

reprint-permission@ams.org.

DOI: http://dx.doi.org/10.1090/noti1666
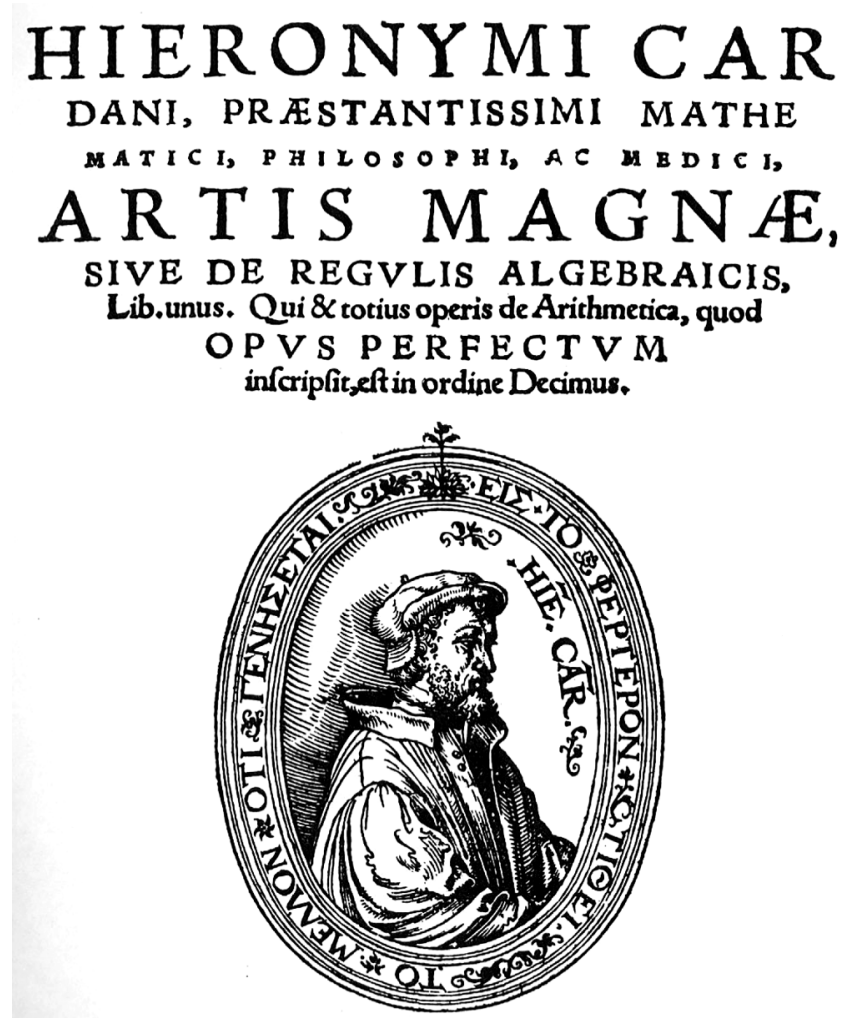

Habes in hoclibro, ftudiofe Lcctor, Regulas Algebraicas ( Itali, de la Cof Hra tocant) nouis adinuentionibus, ac demonftrationibus ab Authore ita locupletatas, ut pro pauculis antea uulgò tritis,iam feptuaginta euaferint. Nes og foltum, ubi unus numerus alteri, aut duo uni, uerum etiam, ubi tuo duobus, aut tres uni ̨qquales furerint, nodum explicant. Hunc aút librumideo feor: fim edere placuit, ut hoc abftrufifsimo, \& plane inexhaufto totius Arithmeti ca thefauro in lucem eruto, $\&$ quafi in theatro quodam omnibus ad fpectan dum expofito, Lectores incitarêtur, ut reliquos Operis Perfectilibros, qui per Tomos edentur, tanto auidius amplectantur, ac minore faftidio perdifcant.

Figure 1. In his 1545 treatise Ars Magna, Gerolamo Cardano introduced imaginary numbers.

Without imaginary numbers, there is no Heisenberg uncertainty principle, no double slit experiment, no Bell's inequality. No reality as we know it.

As my second example, I want to talk about the great Indian mathematician Srinivasa Ramanujan (Figure 2), who was not formally trained in mathematics but made astounding discoveries that dazzle us to this day. He said that many of his formulas were shown to him in his dreams by the family deity, Goddess Namagiri. As soon as he woke up, he would commit them to writing, filling his famous notebooks (the last one was found by George Andrews in 1976 in a box of papers at the Trinity College of Cambridge University). Ramanujan didn't know how to prove most of his marvelous formulas. As G. H. Hardy, who became Ramanujan's mentor and patron, put it, "They must be true because, if they were not true, no one would have had the imagination to invent them" [1]. 

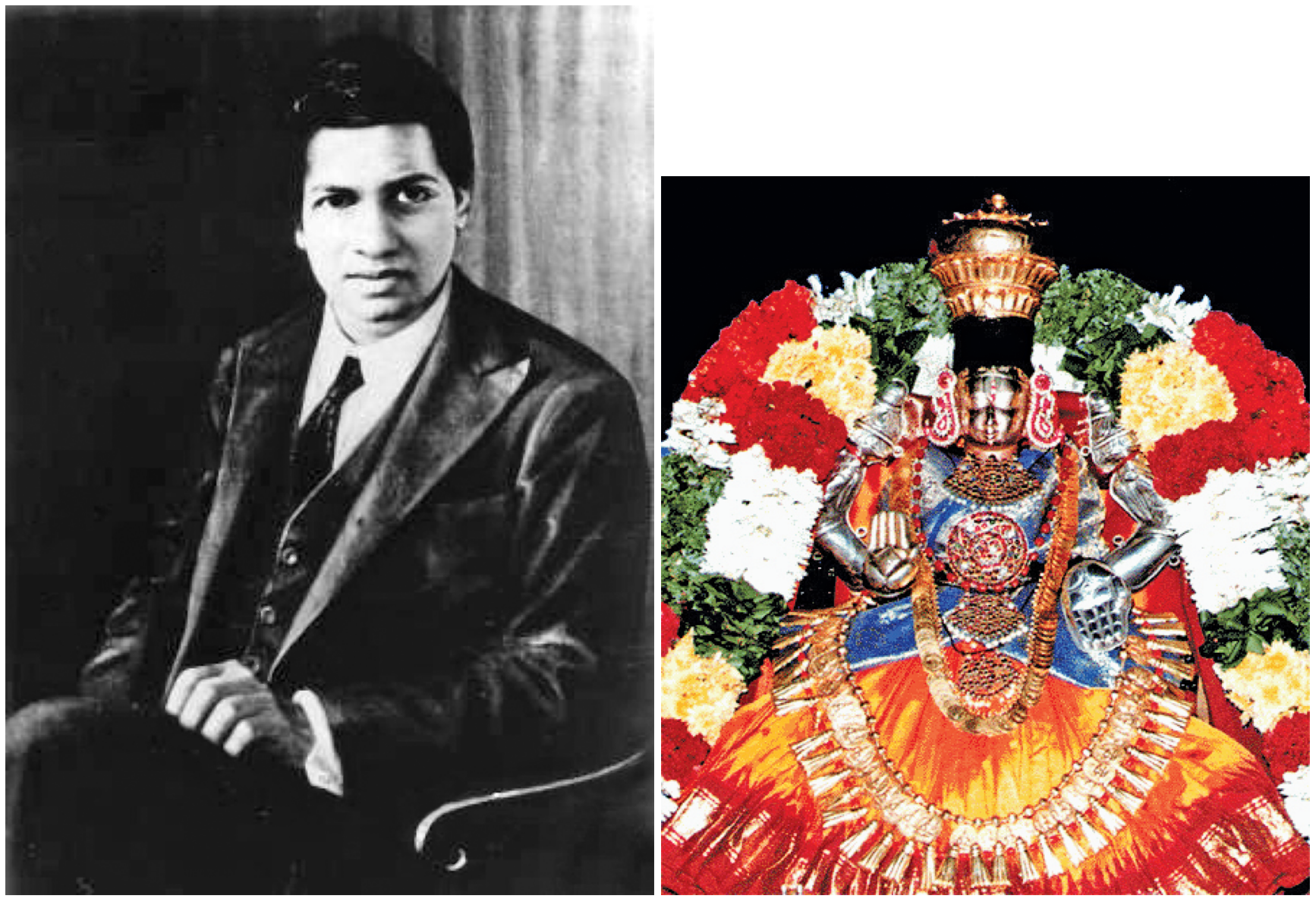

Figure 2. (a) The great Indian mathematician Srinivasa Ramanujan said that many of his formulas were shown to him in his dreams by the family deity, (b) Goddess Namagiri.

But it took several generations of mathematicians to supply the missing proofs.

Perhaps not all mathematicians are visited by a goddess in their dreams, but imagination certainly plays a crucial role in our work. My research is in the Langlands Program, an ambitious project launched by Robert Langlands in the late 1960s aimed at unifying different areas of mathematics. Langlands's original work connected number theory with harmonic analysis in novel and unexpected ways, and in later years similar patterns were found in geometry and even in quantum physics. Studying these ideas, it is quite clear that they were discontinuities in the path of linear knowledge; they could only be brought out by unbridled imagination.

When we look at these examples, a bigger point emerges: a mathematician's mind is not a computer. Indeed, our intuition and imagination cannot be accounted for by computation alone (though, of course, computation plays an essential role in our work). Or, as the great logician Kurt Gödel (Figure 3) convincingly argued in his 1951 Gibbs Lecture to the American Mathematical Society, "The human mind infinitely surpasses the powers of any finite machine." Gödel is echoed by Roger Penrose, who writes, "There is something in our conscious thought process that eludes computation....We have access to mathematical truths that are beyond any robot's capabilities" [2]. It is essential for us mathematicians to appreciate this insight and to share it with others; it sheds light on questions such as, Is life an algorithm?

And that leads us to another lesson: just as mathematicians need to acknowledge, embrace, and utilize their capacity for imagination in order to be successful, so do all humans. Carl Jung [3] warned us that the widening rift between imagination and knowledge (or rather, "what we think we know") is a symptom of "the split consciousness so characteristic of the mental disorders of our day." This split has ushered in a brave new world in which multinational corporations are permitted to derive enormous 


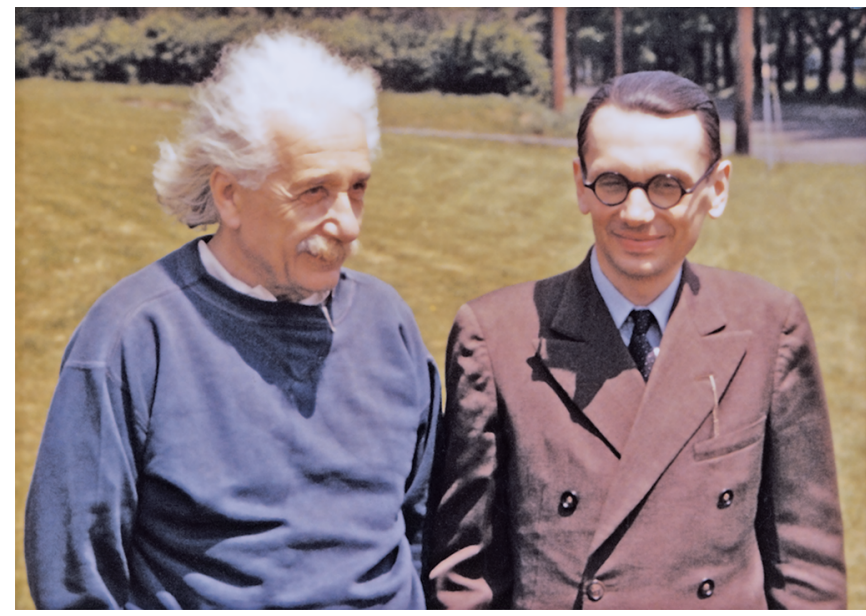

Figure 3. Albert Einstein with Kurt Gödel, who argued that "the human mind infinitely surpasses the powers of any finite machine."

profits from modifying and controlling humans' behavior, while we largely remain silent. In essence, it's the old adage of "divide and conquer"-exploiting our fears, which are always based on what we think we know about ourselves and others-now supercharged with AI-powered information technology. To counter that, we need to use our imagination, which has always been humanity's best antidote to dogma and oppression. Imagination is our way to survive and uphold our dignity. For it's the imagination that gives us a fresh start; it's what reminds us about our common goals and aspirations; it's what unites us. Dare to imagine.

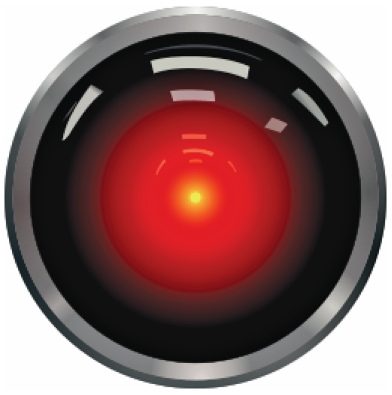

Figure 4. To counter the computer HAL 9000 of Stanley Kubrick's 2001: A Space Odyssey and recent AI-powered information technology, we need to use our imagination. That's what gives birth to the "Star Child" in the film.

\section{References}

[1] G. H. HARDY, The Indian Mathematician Ramanujan, Amer. Math. Monthly 44, (1937), no. 3, 137-155. MR1523880

[2] R. Penrose, Shadows of the Mind, Oxford University Press, 1996. MR1865778

[3] C. Jung, The Undiscovered Self, Routledge, 2013.

\section{Image Credits}

Figure 1 and parts of Srinivasa Ramanujan's (Figure 2) are in the public domain.

Part b of Figure 2: image of Namagiri Thayar from the Anudinam Sri Vaishnava New Portal. "Namakkal Sri Narasimhaswami Temple Navarathri Utsava Patrikai." Uploaded September 25, 2014, by author Mugunthan. Accessed December 29, 2017. anudinam.org/2014/09/25/namakka1-srinarasimhaswami-temple-navarathri-utsava-patrikai.

Figure 3 courtesy of Oskar Morgenstern, photographer. From the Shelby White and Leon Levy Archives Center, Institute for Advanced Study, Princeton, New Jersey.

Figure 4 courtesy of Cryteria (Own work) [CC BY 3.0 (creativecommons.org/1icenses/by/3.0)], via Wikimedia Commons.

Author photo by Emily Scher, courtesy of Edward Frenkel.

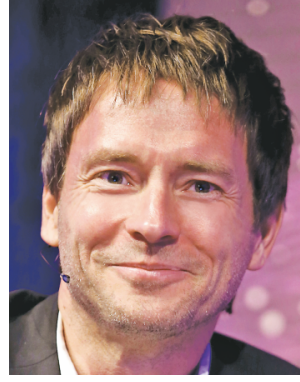

Edward Frenkel

\section{ABOUT THE AUTHOR}

Edward Frenkel is a member of the American Academy of Arts and Sciences, Fellow of the American Mathematical Society, and the winner of the Hermann Weyl Prize in mathematical physics. He is the author of a New York Times bestseller Love and Math, which has been published in eighteen languages. 


\section{Valentino Tosatti}

\section{Metric Limits of Calabi-Yau Manifolds}

The main objects of my talk are the Calabi-Yau manifolds of string theory. Mathematically, these are compact complex manifolds which admit a Kähler metric and whose tangent bundle has vanishing real first Chern class. The simplest examples of Calabi-Yau manifolds are complex tori $\mathbb{C}^{n} / \Lambda$ ( $\Lambda \subset \mathbb{C}^{n}$ a lattice), and when the (complex) dimension is 1 these tori, which are also known as (complex) elliptic curves, are all the examples. In dimension 2 , apart from tori and their finite unramified quotients (known as bielliptic surfaces), one also encounters K3 surfaces, which by definition are simply connected, and their double quotients (Enriques surfaces). These are all the 2-dimensional Calabi-Yaus by the Kodaira-Enriques classification of compact Kähler surfaces (in particular, there are only finitely many topological types). A simple example of a $K 3$ surface is described by the equation $x^{4}+y^{4}=z^{4}+w^{4}$ in $\mathbb{C P}^{3}$, some of whose real points are depicted in Figure 1, and more generally every smooth hypersurface in $\mathbb{C P}^{n+1}$ described by the vanishing of a homogeneous polynomial of degree $n+2$ in $n+2$ variables is a Calabi-Yau $n$-fold.

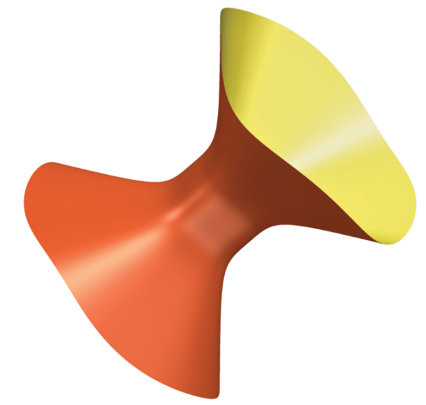

Figure 1 . The $K 3$ surface described by the equation $x^{4}+y^{4}=z^{4}+w^{4}$ in $\mathbb{C P}^{3}$.

Many more examples can be constructed by considering complete intersections in products of projective spaces, and the number of distinct topological types of Calabi-Yau 3 -folds that have been constructed so far is rather large, but it is unknown whether there could be infinitely many.

The reason for the name of these manifolds is Yau's resolution of the Calabi Conjecture: Calabi-Yau manifolds are precisely the compact complex manifolds which admit a Kähler metric whose Ricci curvature is identically zero. These metrics are flat only on tori and their finite quotients. In all the other cases the Riemann curvature tensor does not vanish identically, although its trace (the Ricci curvature) does. Interestingly, these Ricci-flat Kähler

Valentino Tosatti is professor of mathematics at Northwestern University. His email address is tosatti@math . northwestern. edu.

For permission to reprint this article, please contact:

reprint-permission@ams.org.

DOI: http://dx.doi.org/10.1090/noti1661 metrics are not given by an explicit construction (unless they are flat), but instead they are obtained by solving a fully nonlinear second-order PDE of complex MongeAmpère type (involving the determinant of the complex Hessian of a function). For example, nobody knows how to write explicitly a Ricci-flat metric on the $K 3$ surface $x^{4}+y^{4}=z^{4}+w^{4}$ above.

Under mild topological assumptions (simple connectedness and irreducibility), these Ricci-flat metrics have holonomy equal to either $S U(n)$ ("strict Calabi-Yau" manifolds) or $S p\left(\frac{n}{2}\right)$ (hyperkähler or "irreducible holomorphic symplectic manifolds"). The fact that all $K 3$ surfaces admit hyperkähler metrics (note that $S U(2)=S p(1)$ ) is a crucial ingredient to their study. The case of holonomy $S U$ (3) was particularly important in string theory in the 1980 s and led to the development of mirror symmetry for Calabi-Yau 3-folds.

In fact, Yau's theorem gives more precise information: on a given Calabi-Yau manifold with a specified Kähler metric, there is a unique Ricci-flat Kähler metric whose Kähler form is cohomologous to it. In other words, if we fix the complex structure of a Calabi-Yau manifold, the space of all Ricci-flat Kähler metrics on this complex manifold can be identified with the space of all cohomology classes of Kähler forms, the Kähler cone of the manifold. This is an open convex cone in a finite-dimensional vector space. The "metric limits" in the title of the talk refer to the problem of understanding the behavior of families of Ricci-flat Kähler metrics on a Calabi-Yau manifold as we degenerate the cohomology class of their Kähler metrics and/or the underlying complex structure degenerates.

Consider for example the $K 3$ surface $X$ in $\mathbb{C P}^{3}$ described by $x^{4}+y^{4}=z^{4}+w^{4}$, and map a point $[x: y: z: w]$ on $X$ to the complex number $\frac{x^{2}+z^{2}}{w^{2}-y^{2}}=\frac{w^{2}+y^{2}}{x^{2}-z^{2}}$ whenever this is well-defined. This map extends to a surjective holomorphic map $f: X \rightarrow \mathbb{C P}^{1}$ whose fibers are elliptic curves (except for 6 singular fibers). Now consider the family of cohomology classes of Kähler forms $\left[\omega_{t}\right]=$ $\left[f^{*} \omega_{\mathbb{C P}^{1}}\right]+t\left[\omega_{X}\right]$, for $0<t \leq 1$, where $\omega_{\mathbb{C P}^{1}}, \omega_{X}$ are some fixed Kähler forms on $\mathbb{C P}^{1}$ and $X$. By the Calabi-Yau theorem there are Kähler forms $\tilde{\omega}_{t}$ on $X$ in the cohomology class $\left[\omega_{t}\right]$, and these have to degenerate as $t \rightarrow 0$ because the class $\left[f^{*} \omega_{\mathbb{C P} 1}\right]$ does not contain any Kähler form (indeed, its selfintersection vanishes). Understanding the behavior of the Ricci-flat Kähler metrics $\tilde{\omega}_{t}$ as $t \rightarrow 0$ is complicated, because the total volume of these metrics is approaching zero (they are "collapsing") and because of the presence of the singular fibers of $f$. Analytically, the ellipticity of the corresponding family of complex MongeAmpère equations is degenerating everywhere. It was only proved a few years ago in joint work by Mark Gross, Yuguang Zhang, and me that the metrics $\tilde{\omega}_{t}$ converge smoothly away from the singular fibers to the pullback of a Kähler metric with positive Ricci curvature on $\mathbb{C P}^{1}$ minus 6 points, which has mild singularities at these points, and $\left(X, \tilde{\omega}_{t}\right)$ converges in the Gromov-Hausdorff topology (the natural notion of convergence of compact metric spaces) 


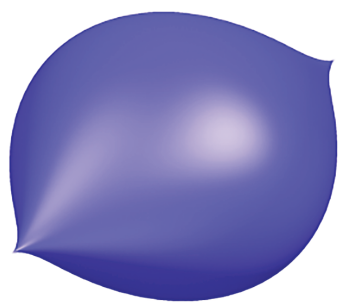

Figure 2. An example of a limiting metric on a 2-dimensional sphere after collapsing of Ricci-flat metrics on a $K 3$ surface. The metric has two singular points corresponding to singular fibers of the elliptic fibration of the $K 3$ surface.

to the completion of this metric. See Figure 2 for an example of such a limiting metric on a 2-dimensional sphere.

In my talk I will explain what we know in general about such metric limits of Ricci-flat Calabi-Yau manifolds, what techniques are used to approach these questions, and what applications these results have.

\section{Image Credits}

Figures 1 and 2 created by the author using free software SURFER, imaginary.org/program/surfer.

Photo of author courtesy of Qiuyu Wang.

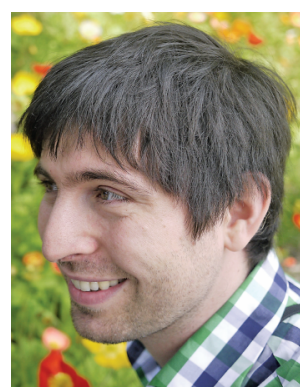

\section{ABOUT THE AUTHOR}

Valentino Tosatti's research lies at the interface between complex and algebraic geometry, geometric analysis, and PDE.

Valentino Tosatti 